\title{
The Golden Section's heptagonal connections
}

\author{
Tomislav Došlić
}

Faculty of Civil Engineering, University of Zagreb

Zagreb, CROATIA

\begin{abstract}
It is well known that the Golden Section plays an important role in the geometry of several polygons and polyhedra; the best known example is the length of a diagonal in the regular pentagon with unit side. In this contribution we show how the Golden Section appears as the solution of an enumerative problem connected with heptagons, more precisely, with heptagonal tilings of the hyperbolic plane. The results are then generalized by investigating whether it also appears in other types of hyperbolic tilings.
\end{abstract}

Keywords: Golden Section, polygonal cluster, heptagon, hyperbolic tiling

MSC: 05A15, 05A16, 52C20

\section{$1 \quad$ Introduction and preliminaries}

The Golden Section, $\phi=\frac{1+\sqrt{5}}{2}$, is, together with its reciprocal value $\varphi=\frac{\sqrt{5}-1}{2}$, one of the most fascinating numbers. Its extraordinary properties and numerous alleged appearances in many wildly different contexts keep attracting attention of mathematicians and laymen alike for more than two and a half millennia. It would go way beyond the scope of this paper to attempt even a far-from-exhaustive enumeration. Instead, we will mention just two of the best known appearances of this number. The first one is geometrical: $\phi$ is the length of a diagonal of a regular pentagon with unit side length. The

DOI: https://doi.org/10.5592/CO/CCD.2020.02 
second one is from enumerative combinatorics, where $\phi$ appears as the limit of ratios of two successive Fibonacci numbers $F_{n+1} / F_{n}$ when $n$ tends to infinity.

The Golden Section often appears in polygons and polyhedra with pentagonal symmetry, such as the icosahedron, sometimes connecting them with (golden) rectangles and triangles. Somewhat less known example is the rhombic triacontahedron all of whose 13 faces are golden rhombi. The number seven is not known for its connections with the Golden Sections. Yet, such connections exist and appear naturally in a context connecting geometry and enumerative combinatorics - that of regular tilings of the (hyperbolic) plane by regular heptagons.

A tiling or a tessellation of a surface is its covering with one or several shapes without gaps and without overlaps. If all shapes are polygons, the tilling is polygonal. A regular tiling is a polygonal tiling in which all polygons have the same number of sides and each vertex is shared by the same number of polygons. In regular tilings, if two tiles share more than one point, then they share a whole edge. The best known regular tilings are the tilings of the Euclidean plane by squares, by regular hexagons and by equilateral triangles shown in Fig. 1.

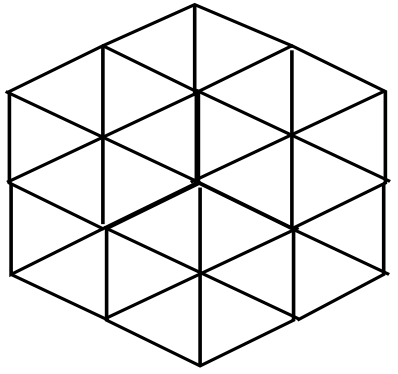

$(3,6)$

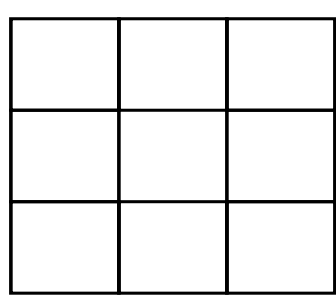

$(4,4)$

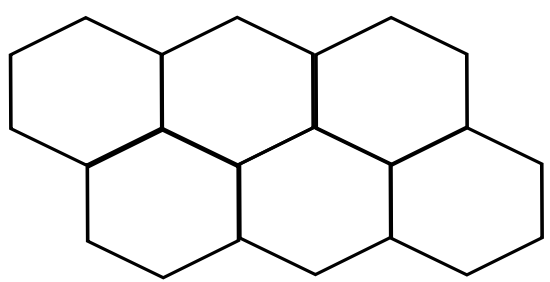

$(6,3)$

Figure 1: The three regular tilings of the Euclidean plane.

Any regular tiling is completely described by two integer parameters $r, s \geq 3$. An $(r, s)$-regular tiling, or simply an $(r, s)$-tiling is the tiling by regular $r$-gons such that exactly $s$ of them meet at every vertex. The same two parameters suffice for classification of regular tilings.

The tilings with $1 / r+1 / s>1 / 2$ cover spherical surfaces. There are only five $(r, s)$ pairs satisfying this condition, and they correspond to the five Platonic solids: $(3,3)$ is the tetrahedron, $(4,3)$ is the 
cube, $(3,4)$ the octahedron, $(5,3)$ is the dodecahedron and $(3,5)$ the icosahedron.

The tilings satisfying $1 / r+1 / s=1 / 2$ are tilings of the Euclidean plane. There are only three of them: $(4,4),(6,3)$ and $(3,6)$ are the familiar tilings of the plane with squares, regular hexagons and equilateral triangles, respectively, mentioned above.

All other regular tilings, i.e., all tilings whose parameters satisfy $1 / r+1 / s<1 / 2$, are tilings of the hyperbolic plane. It is easily seen that there are infinitely many of them. The simplest among them are those with $s=3$, and the smallest value of $r$ yielding a hyperbolic tiling is $r=7$. That brings us to the $(7,3)$-tiling of the hyperbolic plane, the main protagonist of this note.

Another way of looking at tilings is to look at their duals and to define a regular $(r, s)$-tiling as a planar graph in which every vertex is of degree $s$ and every face has $r$ sides. Such graphs can be drawn on the corresponding surface in a regular way, i.e., so that all edges have the same length and all faces the same area; here the lengths and areas are spherical, Euclidean or hyperbolic, depending on the value of $1 / r+1 / s$. We prefer here the face-centered approach since it is easier to count faces than the vertices; see [13] for a vertex-oriented approach. For more on tilings and related matter we refer the reader to the classical monograph by Grünbaum and Shephard [10].

\section{The simplest heptagonal tiling}

In this section we consider the (7,3)-tiling of the hyperbolic plane. We build it iteratively by starting from a single central heptagon and adding successive layers of heptagons. In the first layer there are seven heptagons, each of them sharing an edge with the central heptagon. In the second layer there will be two types of heptagons: Some of them will share one edge with a heptagon in the first layer, while the others will share two adjacent edges, one with each of two adjacent hexagons in the first layer. The situation remains the same for any further layers. The two types of heptagons, denoted as type I and type II, respectively, are shown in Fig. 2. It is easily seen that there are 14 heptagons of type I and 7 heptagons of type II in the second layer, altogether 21 of them.

Let us denote by $f_{n}$ the number of heptagons in the $n$-th layer for $n \geq 0$. Hence $f_{0}=1, f_{1}=7$ and 


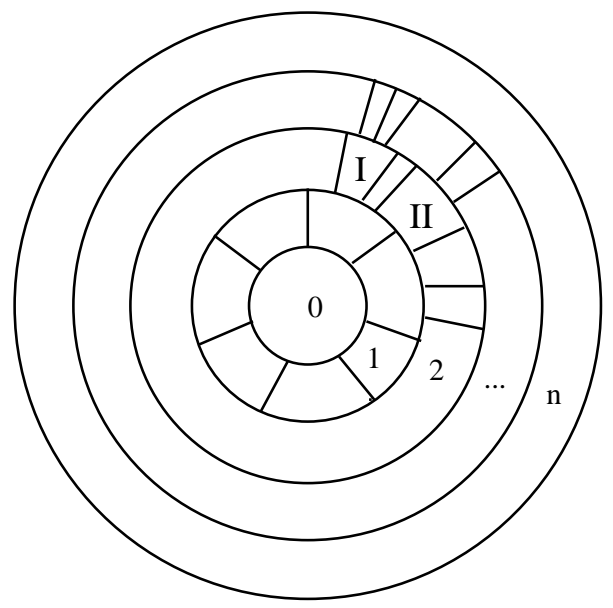

Figure 2: Layers of $(7,3)$-tiling and heptagons of different types.

$f_{2}=21$.

Proposition 1. The number of heptagons in the $n$-th layer of the $(7,3)$-tiling satisfies the recurrence

$$
f_{n}=3 f_{n-1}-f_{n-2} \quad \text { for } \quad n \geq 3
$$

with the initial conditions $f_{1}=7, f_{2}=21$.

Proof. It is clear that each heptagon of the type I from layer $n-1$ sends out three radial edges to the layer $n$, while each heptagon of type II sends out only two radial edges. Hence the number of outgoing radial edges can be obtained by multiplying the total number of heptagons in the $(n-1)$-st layer by 3 and then subtracting the number of heptagons of type II. As there are exactly $f_{n-2}$ heptagons of type II in the $(n-1)$-st layer, the total number of outgoing radial edges is given by $3 f_{n-1}-f_{n-2}$. But this is exactly the number of heptagons in the $n$-th layer, and that proves the claim.

It follows immediately that the numbers $f_{n}$ are multiples of even-indexed Fibonacci numbers.

Corollary 2. $f_{n}=7 F_{2 n}$ for $n \geq 1$. Here $F_{2 n}$ are the even-indexed Fibonacci numbers.

Now we can easily compute the total number of heptagons in the first $n$ layers of a $(7,3)$-tiling. If we denote this quantity by $g_{n}$, we have $g_{n}=\sum_{k=0}^{n} f_{k}$. By using Corollary 2 and the well-known formula $\sum_{k=1}^{n} F_{2 k}=F_{2 n+1}-1$, we immediately obtain

$$
g_{n}=1+7 \sum_{k=1}^{n} F_{2 k}=7 F_{2 n+1}-6 .
$$


So, the total number of heptagons in the first $n$ layers of a $(7,3)$-tiling has a neat expression in terms of odd-indexed Fibonacci numbers.

The iterative constructions of the described type can serve as useful models of certain growth processes not only in the hyperbolic, but also in the Euclidean space. A nice example is presented by leaves and flowers of certain vegetables, which can be also mimicked by crocheting; see [14] for several nice illustrations. In such situations it might be of interest to consider the fraction of the periphery, whether expressed in terms of the number of cells, of the length of yarn, or of the mass of tissue, in the total bulk of the structure. It is known that in the Euclidean case this fraction tends to zero, since the total number of cells grows quadratically, while the number of peripheral cells grows only linearly with the number of generations (i.e., of layers). In the hyperbolic case the growth is exponential, and hence the fraction of peripheral cells becomes non-negligible. In the case of the (7,3)-tiling, the fraction of peripheral cells can be expressed in a particularly simple and elegant way.

Corollary 3. The fraction of peripheral heptagons in a $(7,3)$-tiling tends to $\varphi=\frac{\sqrt{5}-1}{2}$ for large values of $n$.

This is a direct consequence of Corollary 2 and of the already mentioned fact that the ratio of two successive Fibonacci numbers tends to the Golden Section for large values of $n$. So, there is the connection promised in the title of this note.

It remains to examine whether heptagons are in some respect special or the same fraction of periphery can also appear in some other tilings.

\section{$3 \quad(r, 3)$-tilings}

In this section we look at regular tilings of the hyperbolic plane with regular $r$-gons, such that exactly 3 of them meet at every vertex. Again, we start with a single $r$-gon in the center surrounded by $r$-gons in the first layer. The number of $r$-gons in the $n$-th layer we denote by $p_{n}$. As the cases $r=3,4,5$ lead to the Platonic solids, we consider only $r \geq 6$.

Proposition 4. The numbers $p_{n}$ satisfy the recurrence $p_{n}=(r-4) p_{n-1}-p_{n-2}$ for $n \geq 3$ with the initial conditions $p_{1}=r, p_{2}=(r-4) r$. 
The proof follows along the same lines as the proof for the heptagonal case in the previous Section, so we omit the details.

It is a routine exercise to compute the generating functions for sequences $p_{n}$ and for sequences of their partial sums $q_{n}=\sum_{k=0}^{n} p_{k}$. Again, we omit the details and state only the final expressions.

$$
P(x)=\sum_{n=0}^{\infty} p_{n} x^{n}=\frac{1+4 x+x^{2}}{1-(q-4) x+x^{2}}, \quad Q(x)=\sum_{n=0}^{\infty} q_{n} x^{n}=\frac{1+4 x+x^{2}}{(1-x)\left(1-(q-4) x+x^{2}\right)} .
$$

From the generating functions we can determine the asymptotic behavior of the enumerating sequences and then use it to compute the fraction of $r$-gons in the peripheral layer. We refer the reader to $[1,15]$ for more information on obtaining the asymptotics of a sequence from its generating function. Here we rely on the following version of Darboux's theorem [1].

\section{Theorem A}

If the generating function $f(x)=\sum_{n \geq 0} a_{n} x^{n}$ of a sequence $\left(a_{n}\right)$ can be written in the form $f(x)=$ $\left(1-\frac{x}{w}\right)^{\alpha} h(x)$, where $w$ is the smallest modulus singularity of $f$ and function $h$ is analytic at $w$, then $a_{n} \sim \frac{h(w) n^{-\alpha-1}}{\Gamma(-\alpha) w^{n}}$, where $\Gamma$ denotes the gamma function.

Since both our generating functions $P(x)$ i $Q(x)$ have the same smallest modulus singularity $w=$ $w(r)=\frac{1}{2}\left(r-4-\sqrt{(r-4)^{2}-4}\right)$, both $p_{n}$ and $q_{n}$ will asymptotically behave as $w(r)^{n}$ and there will be a lot of cancellation in the ratio $p_{n} / q_{n}$. In fact, the only surviving term is $1-w(r)$. As $w(r)$ is a decreasing function of $r$, tending to zero for large values of $r$, it follows that the fraction of peripheral $r$-gons tends to one for large values of $r$ and $n$.

Proposition 5. The fraction of peripheral $r$-gons in an $(r, 3)$-tiling is $1-w(r)$ and it tends to one for large values of $r$ and $n$.

Hence for large values of $r$ almost all polygons are on the periphery. That offers an explanation for patterns observed in some living organisms employing such arrangements as an efficient way to package many parts which must be in contact with each other and also with the surroundings.

For $r=7$ we have $w(7)=\frac{1}{2}(3-\sqrt{5})=\phi^{-2}=\varphi^{2}$. Since $\varphi=1-\varphi^{2}$, the case $r=7$ is the only one yielding the Golden Section as the fraction of peripheral $r$-gons. 
We close this section by observation that in the case $r=6$ we obtain $w(6)=1$, thus confirming the non-exponential nature of the growth of $p_{n}$ and $q_{n}$.

\section{$4 \quad(r, s)$-tilings}

Finally we consider the most general case $s>3$. The case $r=3$ will need a separate treatment, so assume, for the beginning, $r \geq 4$.

Let $z_{n}=z_{n}(r, s)$ denote he number of $r$-gons in the $n$-th layer of a regular $(r, s)$-tiling. As before, we have one central $r$-gon, so $z_{0}=1$. In the first layer there will be one $r$-gon for each of $r$ sides of the central $r$-gon and $s-3 r$-gons for each of its $r$ vertices. Hence, $z_{1}=r(s-2)$.

In the second layer, and in all further layers, there will be two types of $r$-gons - the ones sharing a whole edge with $(n-1)$-st layer, and the ones sharing only one vertex. We call them type I and type II, respectively, and denote their respective numbers in the $n$-th layer by $v_{n}$ and $w_{n}$.

Clearly, each of the $v_{n} r$-gons of type I has one of its sides on the interior border of the $n$-th layer; it shares two of its sides with other $r$-gons of the same layer, and hence it has $r-3$ sides with $r-4$ vertices of degree 2 on the outer border of the $n$-th layer. Similarly, each of the $w_{n} r$-gons of type II shares two sides with other $r$-gons of the $n$-th layer and has $r-2$ sides with $r-3$ vertices on the outer border. There are altogether $v_{n}+w_{n}$ vertices of degree 3 on the outer border of the $n$-th layer.

Now we can compute the number of $r$-gons of each type in the next layer, as each side on the outer border gives rise to one $r$-gon of type I, and each vertex on the outer border gives rise to $s-3$ or $s-4$ $r$-gons of type II, depending on its degree. See Fig. 3. Hence,

$v_{n+1}=(r-3) v_{n}+(r-2) w_{n}$

$w_{n+1}=(s-3)\left[(r-4) v_{n}+(r-3) w_{n}\right]+(s-4)\left(v_{n}+w_{n}\right)$.

After some rearrangement, we obtain a double linear recurrence

$$
\begin{aligned}
v_{n+1} & =(r-3) v_{n}+(r-2) w_{n} \\
w_{n+1} & =[(s-3)(r-4)+(s-4)] v_{n}+[(s-3)(r-3)+(s-4)] w_{n}
\end{aligned}
$$

for $n \geq 2$ with the initial conditions $v_{1}=r, w_{1}=r(s-3)$. (Essentially the same recurrence appears 

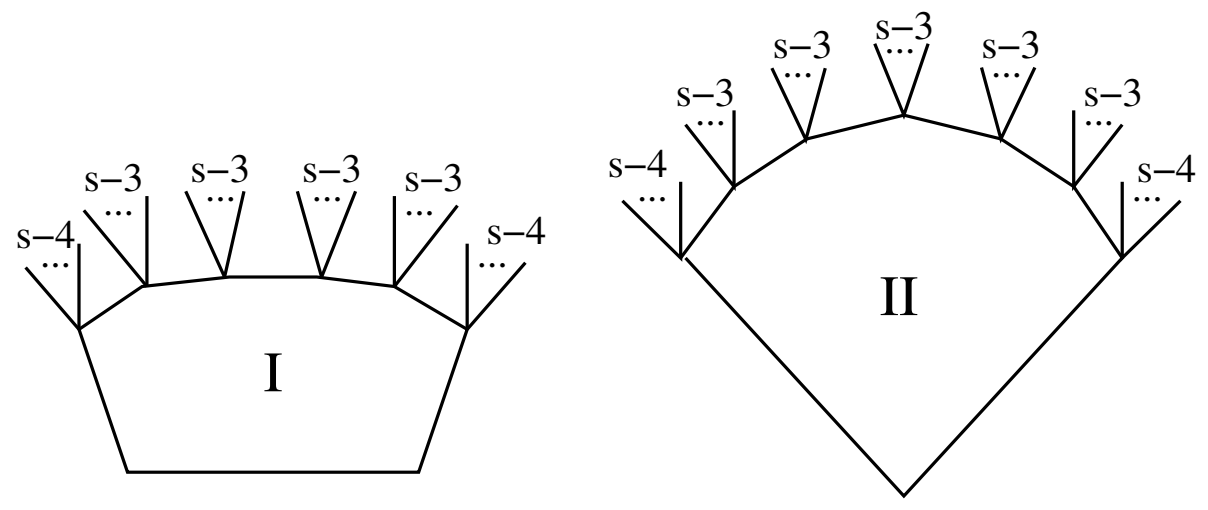

Figure 3: Two types of $r$-gons in a regular $(r, s)$-tiling.

in Lemma 1 of ref. [12] concerned with some probabilistic aspects of certain trees related to regular hyperbolic tilings.) One could proceed by expressing $w_{n}$ from the first recurrence, plug it into the second one and solve the resulting second-order linear recurrence for $w_{n}$. Instead, we consider a general first-order linear double recurrence with constant coefficients

$$
\begin{aligned}
v_{n+1} & =A v_{n}+B w_{n} \\
w_{n+1} & =C v_{n}+D w_{n}
\end{aligned}
$$

for $n \geq 2$ with the initial conditions $v_{1}=E, w_{1}=F$. Let $V(x)$ and $W(x)$ be the generating functions of sequences $v_{n}$ and $w_{n}$, respectively. It is a straightforward exercise in generating function manipulation to convert the above recurrence into a linear system for $V(x)$ and $W(x)$,

$$
\begin{aligned}
V(x) & =A x(V(x)-E)+B x(W(x)-F) \\
W(x) & =C x(V(x)-E)+D x(W(x)-F)
\end{aligned}
$$

which can be easily solved by using Cramer's rule. The resulting expression for $Z(x)=V(x)+W(x)$ is

$$
Z(x)=x \frac{(A D-B C)(E+F) x-[(A+C) E+(B+D) F]}{1-(A+D) x+(A D-B C) x^{2}} .
$$

By plugging in $A D-B C=1$ and $A+D=r s-2(r+s-1)$ and by accounting for the index shift, we finally obtain

$$
Z(x)=\frac{1+2(s-1) x+x^{2}}{1-[r s-2(r+s-1)] x+x^{2}},
$$

corresponding to the recurrence

$$
z_{n}=[r s-2(r+s-1)] z_{n-1}-z_{n-2}
$$


for $z_{n}$ with the initial conditions $z_{0}=1, z_{1}=r(s-2), z_{2}=r(s-2)[r s-2(r+s-1)]$.

It remains to consider the case $r=3$. We may assume $s \geq 6$, since the smaller values of $s$ lead to Platonic solids. Let $t_{n}$ denote the number of triangles in the $n$-th layer. As before, $t_{0}=1, t_{1}=3(s-2)$ and $t_{2}=3(s-2)(s-4)$. Let $u_{n}$ denote the number of vertices on the outer border of the $n$-th layer. Some of them are of degree 3 , some are of degree 4 . Those of degree 3 send $s-3$ radial edges out to the $(n+1)$-st layer; the ones of degree 4 send out $s-4$ radial edges. Clearly, the number of vertices of degree 4 is equal to the number of all vertices on the inner border of the considered layer, hence to $u_{n-1}$. Now the total number of outgoing radial edges can be computed as $(s-3) u_{n}-u_{n-1}$. Since each of $u_{n}$ pairs of adjacent vertices sends out two edges that meet in a vertex on the outer border

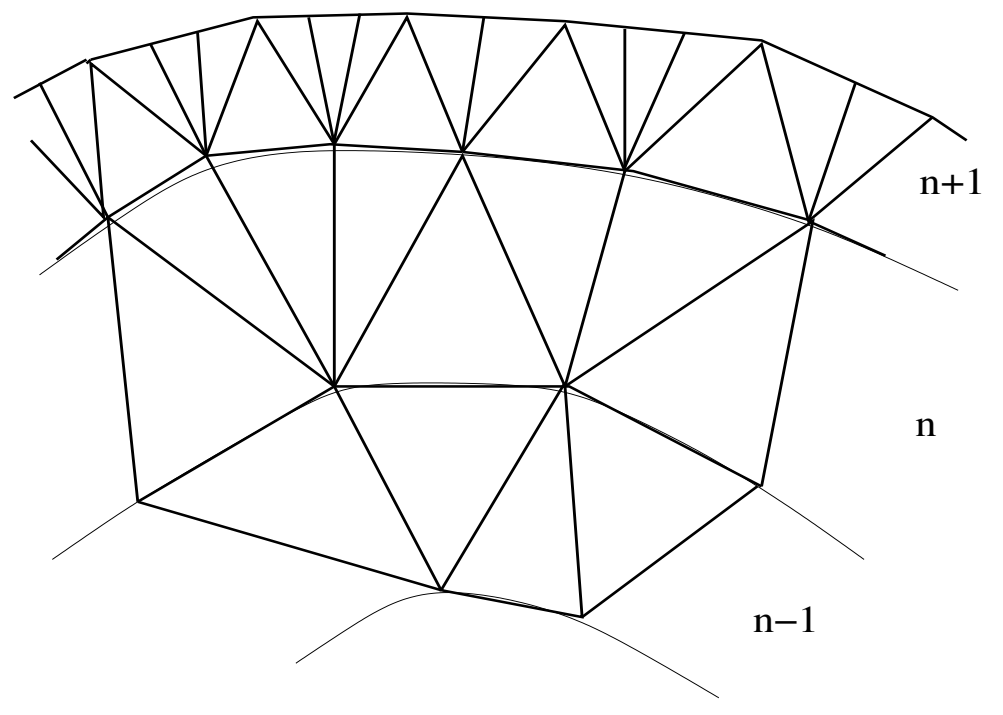

Figure 4: Fragments of three successive layers in a $(3,7)$-tiling.

of the $(n+1)$-st layer, we must subtract $u_{n}$ from the total number of outgoing radial edges in order to obtain $u_{n+1}$. Hence, $u_{n+1}=(s-4) u_{n}-u_{n-1}$. The situation is illustrated in Fig. 4 . Now it immediately follows that $t_{n}=u_{n}+u_{n-1}$, since all triangles in the $n$-th layer share two of their sides with other triangles in the same layer. Those with the remaining side on the inner border are counted by $u_{n-1}$, while those with the third side on the outer border are counted by $u_{n}$. As both $u_{n}$ and $u_{n-1}$ satisfy the same recurrence, their sum must satisfy the same recurrence, with suitably modified initial conditions. Hence, $t_{n}=(s-4) t_{n-1}-t_{n-2}$ for $n \geq 3$ with $t_{1}=3(s-2)$ and $t_{2}=3(s-2)(s-4)$.

We see that the $(3, s)$-tilings follow the same recurrence as the tilings with polygons with larger number of sides. Further, for $s=6$ we recover the polynomial growth of the tilings of Euclidean plane. It is 
interesting to note that our reasoning remains, in a sense, valid also for $s=5$ : from $t_{1}=9$ and $t_{2}=9$ one obtains $t_{3}=0$; that is wrong as the number of triangles in the third layer (equal to 1 ), but correct as the number of outgoing edges.

Now we can summarize our findings on $(r, s)$-tilings.

Proposition 6. The number $z_{n}$ of $r$-gons in the $n$-th layer of a regular $(r, s)$-tiling satisfies the recurrence

$$
z_{n}=[r s-2(r+s-1)] z_{n-1}-z_{n-2}
$$

for $n \geq 3$ with the initial conditions $z_{0}=1, z_{1}=r(s-2), z_{2}=r(s-2)[r s-2(r+s-1)]$.

It remains to check if any of $(r, s)$-tilings for $s>3$ have the growth rate resulting in the Golden Section fraction of peripheral polygons. As $\varphi^{-2}$ is the only $w=w(r, s)$ such that $1-w=\varphi$, we must look for integer solutions of the equation $r s-2(r+s-1)=3$, or, equivalently, $r s=2(r+s)+1$, satisfying the additional condition $r, s \geq 3$. Clearly, $r=\frac{2 s+1}{s-1}<3$ for all $r \geq 3, s \geq 8$. That leaves only the case $s=7$, and it is easy to check that $r=3$ is the only case satisfying the equation. Hence, we have identified all $(r, s)$-tilings in which the fraction of peripheral polygons tends to $\varphi$ for large $n$.

Theorem 7. There are only two regular $(r, s)$-tilings such that the fraction of peripheral polygons tends to the reciprocal value of the Golden Section. Those two tilings are the regular $(7,3)$ and $(3,7)$ tilings of the hyperbolic plane.

We close this section by mentioning that many regular $(r, s)$-tilings have growth rates that are positive integer powers of $\phi$. It has been known for a long time [2] that the polynomials $x^{2}-L_{n} x+(-1)^{n}$ have the roots $\phi^{n}$ and $(-\varphi)^{n}$. (Here $L_{n}$ denotes the $n$-th Lucas number.) For example, the case $n=4$ yields the polynomial $x^{2}-7 x+1$ with the roots $\phi^{4}$ and $\varphi^{4}$ that arises, rather trivially, as the characteristic polynomial of recurrences for the number of polygons in the $n$-th layer of a dual pair of regular $(11,3)$ and $(3,11)$ tilings, and also, somewhat less trivially, for the $(5,5)$-tiling. However, only the case $n=2$ results in the fraction of peripheral polygons which stands in a simple relation to the Golden Section. We leave to the interested reader to investigate for what other regular $(r, s)$-tilings their growth rates are positive integer powers of the Golden Section. 


\section{Concluding remarks}

In this contribution we have explored some properties of regular hyperbolic tilings and identified all cases where the Golden Section appears as the fraction of peripheral cells in the bulk of a tiling. We found that there are only two such tilings, the dual pair $(7,3)$ and $(3,7)$. In all other possible cases the fraction of periphery in the total exceeds $\varphi$. Our intention was to point out an appearance of the Golden Section in a geometric context outside its usual habitat of polygons and polyhedra with pentagonal symmetry. Although it seems that heptagons and triangles play equally important roles in our findings, we have chosen to emphasize the heptagonal connections since the triangular ones are far better known. We leave to the interested reader to decide whether the fact that the number five lies halfway between three and seven has any relevance for our findings.

The results presented in this note are certainly not new. Most likely they were not new even back in 2003 when I first obtained them as a spin-off from a paper concerned with certain types of fullerene graphs. Since then, several papers appeared addressing some related but more general problems $[4,6,13]$, and most of the present results can be obtained by specializing certain parameters in the main results of references [7-9]. However, the results are not widely known either - only a handful of the enumerating sequences are present in the On-Line Encyclopedia of Integer Sequences, and mostly without references to the corresponding tessellations. Croatian readers might find it interesting that the study of the objects considered here seems to be initiated by S. Bilinski in his Ph. D. thesis [3].

I am aware of one more heptagonal connection of the Golden Section: A closely related quantity,

$\sqrt{5}=\phi+\varphi$, appears as the limit of ratios of certain quantities relevant for the study of sandpile models on the regular heptagonal tiling [11]. An interested reader might wish to explore whether the heptagonal tiling is the only regular hyperbolic tiling with this property.

\section{References}

[1] E. A. Bender, S. Gill Williamson, Foundations of Combinatorics with Applications, Dover, 2006.

[2] M. Bicknell, N. A. Draim, Sum of $n$-th power of roots of a given quadratic equation, Fibonacci 
Quart. 4.2 (1966) 170-178.

[3] S. Bilinski, Homogene mreže ravnine, Rad JAZU 271 (1948) 145-255.

[4] J. A. Bruce, M. E. Watkins, Concentric Bilinski diagrams, Australas. J. Combin. 30 (2004) $161-174$.

[5] J. W. Cannon, The combinatorial structure of cocompact discrete hyperbolic groups, Geom. Dedicata 16 (1984) 123-148.

[6] J. Choi, N. Pippenger, Counting the Angels and Devils in Escher's Circle Limit IV, J. Humanistic Math. 5 (2015) 51-59.

[7] S. J. Graves, Tessellations with arbitrary growth rates, Discrete Math. 310 (2010) 2435-2439.

[8] S. Graves, T. Pisanski, M. E. Watkins, Growth of edge-homogeneous tessellations, SIAM J. Discrete Math. 23 (2008) 1-18.

[9] S. J. Graves, M. E. Watkins, Growth of face-homogeneous tessellations, Ars Math. Contemp. 14 (2018) 285-318.

[10] B. Grünbaum, G. C. Shephard, Tilings and Patterns, W. H. Freeman and Co., New York, 1987.

[11] N. Kalinin, M. Shkolnikov, Sandpiles on the heptagonal tiling, J. Knot Theory Ramif. 25 (2016) 1642005.

[12] L. Németh, Trees on hyperbolic honeycombs, Miskolc Math. Notes 16 (2015) 353-360.

[13] A. Paul, N. Pippenger, A census of vertices by generations in regular tessellations of the plane, Electronic J. Combin. 18 (2011) \#R87.

[14] M. Trnkova, Hyperbolic flowers, arXiv:1910.05900v1.

[15] H. S. Wilf, generatingfunctionology, AK Peters, Wellesley, MA, 2006. 